\title{
BMJ Open HORIZONS protocol: a UK prospective cohort study to explore recovery of health and well-being in adults diagnosed with cancer
}

\author{
Claire Foster, ${ }^{1}$ Lynn Calman, ${ }^{1}$ Alison Richardson, ${ }^{1}$ Carl R May, ${ }^{2}$ Anne Rogers, ${ }^{1}$ \\ Peter W Smith ${ }^{3}$
}

To cite: Foster C, Calman L, Richardson A, et al. HORIZONS protocol: a UK prospective cohort study to explore recovery of health and wellbeing in adults diagnosed with cancer. BMJ Open 2019;9:e029662. doi:10.1136/ bmjopen-2019-029662

- Prepublication history and additional material for this paper are available online. To view these files, please visit the journal online (http://dx.doi. org/10.1136/bmjopen-2019029662).

Received 4 February 2019 Revised 22 June 2019 Accepted 24 June 2019

Check for updates

(c) Author(s) (or their employer(s)) 2019. Re-use permitted under CC BY-NC. No commercial re-use. See rights and permissions. Published by BMJ.

${ }^{1}$ School of Health Sciences, University of Southampton,

Southampton, UK

${ }^{2}$ Faculty of Epidemiology and Population Health, London

School of Hygiene and Tropical

Medicine, London, UK

${ }^{3}$ Social Statistics and

Demography, University of

Southampton, Southampton, UK

Correspondence to

Professor Claire Foster;

C.L.Foster@soton.ac.uk

\section{ABSTRACT}

Introduction Understanding the impact of cancer and its treatment on people's everyday lives will help prepare people for what to expect, enable health professionals to predict likely recovery trajectories and shape care management according to needs. HORIZONS will recruit people awaiting treatment and follow them up at regular intervals to assess recovery of health and well-being. Research questions What impact does cancer diagnosis and treatment have on people's lives in the short, medium and long term? What are people's health and well-being outcomes, experiences and selfmanagement activities over time across different cancer types and what influences these? How do people connect with and relate to others in mobilising resources that enable them to self-manage the consequences of cancer and treatment?

Methods and analysis HORIZONS is a multicentre, prospective cohort study exploring recovery of health and well-being in 3000 people diagnosed with breast cancer ( $<50$ years), non-Hodgkin's lymphoma or gynaecological cancer. Recruitment will take place across National Health Service (NHS) sites in the UK between September 2016 and March 2019, before primary treatment starts. Participants will be identified through clinical teams and invited to complete questionnaires including assessments of quality of life, symptoms and functioning (Quality of Life in Adult Cancer Survivors; European Organisation for Research and Treatment Consortium Core quality of life questionnaire, EORTC-QLQ-C30), health status (EuroQol-5 dimensions, EQ-5D), self-efficacy, social support, social networks and lifestyle. Clinical data will also be collected. Descriptive statistics will characterise outcomes. Changes over time will be investigated. Factors that may influence recovery and self-management will be included in regression models to determine which influence health and well-being and self-management.

Ethics and dissemination Ethics and Health Research Authority approvals granted (IRAS Project ID: 202342, REC reference number 16/NW/0425). Adopted onto the National Institute for Health Research Clinical Research Network portfolio. We will engage with our Scientific Advisory Board, Tumour Specific Expert Panels, User Reference Group, Macmillan and the University of Southampton to ensure maximum publicity and benefit.
Strengths and limitations of this study

- A combination of patient-reported outcomes and clinical data from medical notes.

- Completion of patient-reported outcome measures prior to treatment commencing.

- Total sample identified and approached wherever possible in multiple sites across the UK.

- Collection of data on a range of theoretically informed variables to understand the wider impact of cancer and treatment on everyday lives.

- Not possible to collect baseline measures of health and well-being prior to cancer diagnosis or recruit a comparator cohort, however, specific outcome measures are included to allow for comparisons to existing UK Iongitudinal population studies.

\section{INTRODUCTION}

Around 13 million people worldwide are diagnosed with cancer every year. This figure is expected to triple by 2050 . The number of people living with and beyond cancer in the UK is expected to reach four million by $2030 .{ }^{1}$ Healthcare systems need to adapt to manage this demand and better meet the needs of this growing population. Global initiatives are exploring ways to improve care for cancer survivors and calling for high-quality interdisciplinary research to provide evidence to inform the most effective ways to support survivors to improve health and well-being. ${ }^{2}$

Benefits can be experienced following a cancer diagnosis; however, many people face challenges that can have a major impact on all aspects of daily life. ${ }^{3}$ The need for support in managing this has been highlighted. ${ }^{4}$ People can be unprepared for the impact on their lives, may feel vulnerable, experience loss of confidence, struggle to manage and access care and support. ${ }^{35-7}$ This in turn may have implications for recovery, self-management and timely access to appropriate services. 
In these respects, the current healthcare system is not adequately meeting the needs of people after cancer treatment. ${ }^{8}$

In order to address future needs appropriately it is necessary to understand how people live with and manage the consequences of cancer and its treatment so that they can be supported optimally, consider their ability to manage this and the implications for their personal networks in supporting them. Increasingly, clinical trials collect quality of life (QoL) data. However, clinical trials are applicable to a minority of patients and have high dropout rates ${ }^{9}$ and QoL assessments alone do not give a full picture of the impact of cancer and treatment on everyday life or how people manage. A number of initiatives have collected regional or national patient-reported psychosocial outcomes including QoL data to investigate the wider aspects of recovery following a cancer diagnosis and treatment. These include cross-sectional studies providing a snapshot of recovery in time ${ }^{10-12}$ longitudinal studies monitoring changes over time ${ }^{813}$ and trials of psychosocial interventions. ${ }^{14}$ None of these, however, combine a longitudinal design with measurement of outcomes before and after the start of treatment which would allow better understanding of the consequences of cancer treatment on recovery.

HORIZONS will improve our understanding of recovery following curative intent treatment for cancer, providing in-depth and wide-ranging evidence. HORIZONS uses a prospective, cohort approach to gather patient reported and clinical data, involving representative groups of people recently diagnosed with cancer to reveal how the consequences of cancer and its treatment affect their lives, how this is managed in everyday life, how this changes with time and what influences this.
HORIZONS will provide comprehensive data needed by patients, members of their social networks, clinical and policy communities to inform the transformation to personalised care for people living with and beyond cancer.

HORIZONS is underpinned by a conceptual framework (see figure 1) informed by three areas of work:

1. Foster and Fenlon's ${ }^{3}$ conceptual framework of recovery of health and well-being following cancer treatment hypothesises that health and well-being are positively associated with presence of support for the individual, their confidence to manage problems faced as a consequence of their cancer and negatively associated with problems experienced such as fatigue and pain. ${ }^{15}$

2. Burden of treatment theory ${ }^{2}$ seeks to understand how people self-manage the demands placed on them by cancer, its treatment and the healthcare system, their capacity to do this work and individual/group mechanisms that promote or inhibit development of capacity for self-management.

3. A social network analytical approach will enable us to explore self-management support in survivorship conceptualising personal networks as a source from which resources can be mobilised and used and the types of illness 'work' undertaken within peoples' social networks which contribute to personal coping and management. ${ }^{16}{ }^{17} \mathrm{~A}$ major source of support can come from personal networks. Social network members are recognised as having an important role in self-management. ${ }^{16}$

The study is funded by Macmillan Cancer Support a UK charity that supports people living with and beyond cancer by providing information, support, services and funding research.

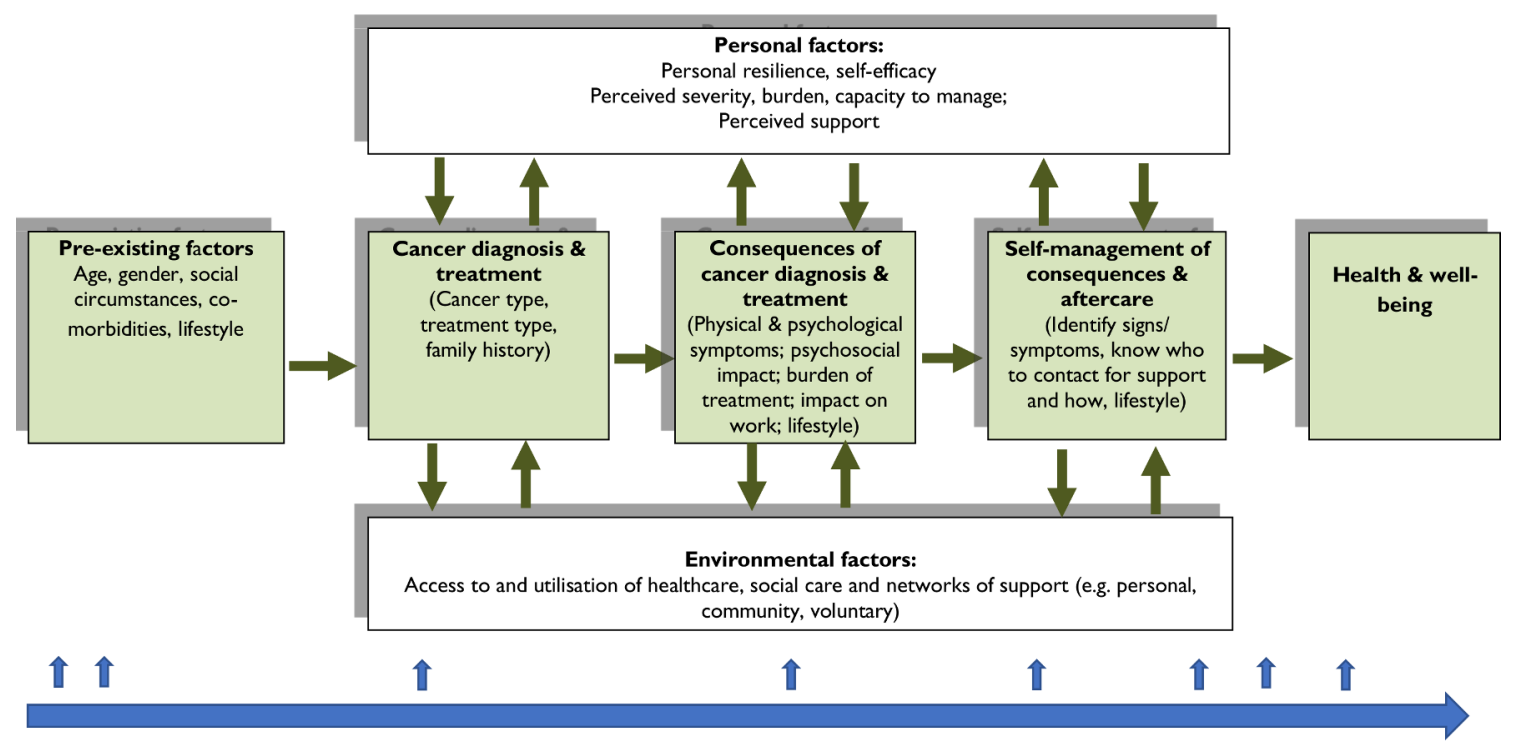

Life-course (expected and unexpected events through the life-course e.g. co-morbidities, recurrence, births, marriages, deaths, moving to a new house, new job, retirement, increasing frailty)

Figure 1 Conceptual framework underpinning the HORIZONS programme (informed by Foster and Fenlon, ${ }^{3}$ May et al, ${ }^{2}$ Vassilev et $a^{16}{ }^{17}$ ) 


\section{METHODS AND ANALYSIS}

A series of longitudinal cohorts will be established. Participants will complete self-report questionnaires at regular intervals and clinical data will be collected. A nested qualitative study will be developed to understand, characterise and explain how people connect with and mobilise supportive services and resources to help them to selfmanage the consequences of cancer and its treatment and to promote recovery of health and well-being. The study design will be developed during the first 2 years of the HORIZONS programme and submitted for ethical approval as an amendment to this protocol.

Activity will be structured around two linked research themes.

1. Recovery from cancer treatment: to explore determinants of recovery following primary cancer treatment. Our hypothesis is that curative cancer treatment disrupts health and well-being and people require time and support to recover. We will explore patterns of physical, psychological, social functioning and what helps or hinders this to characterise trajectories of recovery. We will also explore who is most at risk of a protracted or complex recovery pathway.

2. Self-managing the consequences of cancer: to examine how cancer and its consequences are managed and how management changes over time. Our hypothesis is that consequences of cancer and its treatment disrupt ways of managing, irrespective of level of health need, although those with complex needs will need most support. Those who feel supported to self-manage will be more able to self-manage. We will also determine the work involved in self-management, who is most likely to need support to self-manage, what helps/hinders this, when support should be offered and in what form. It will also allow the identification of those who are least likely to self-manage successfully.

\section{Research questions}

1. What impact does cancer diagnosis and treatment have on people's lives in the short, medium and long term?

2. What are people's health and well-being outcomes, experiences and self-management activities over time across different cancer types and what influences these?

3. How do people connect with and relate to others in mobilising resources that enable them to self-manage the consequences of cancer and treatment?

\section{Patient and public involvement}

Patient and public involvement (PPI) in HORIZONS began during the development of the study protocol and supporting documents. PPI representatives will contribute to the design of study outcome measures (questionnaires and case report forms (CRFs)) and other patient facing documentation including information sheets, invitation letters and patient newsletters. They will comment on recruitment methods, ways to improve study retention and the conduct of the qualitative study. The
HORIZONS User Reference Group will meet regularly at the University of Southampton. PPI representatives will sit on the Strategic Advisory Board and Tumour Specific Expert Panels.

\section{Study setting}

All patients meeting the eligibility criteria will be identified and recruited through their clinical teams prior to primary treatment. The study will be managed from the University of Southampton through the HORIZONS Coordinating Centre. HORIZONS has been adopted onto the NIHR CRN Portfolio. Participants will be identified and recruited from NHS treatment centres across the UK. Around 70 NHS Trusts and Health Boards will be selected covering a wide range of geographical locations across the UK, ethnically diverse populations and varying-sized hospitals.

\section{Participants}

Patients with the following cancers will be invited to participate: breast cancer diagnosed under age 50 years; non-Hodgkin's lymphoma (NHL) (diffuse large B cell subtype: DLBCL); gynaecological cancers (endometrial, cervical, ovarian and vulval). Generic inclusion/exclusion criteria are below; see online supplementary appendix 1 for specific requirements per cancer type.

The cohorts were chosen in collaboration with Macmillan Cancer Support's Clinical Advisory Board. They were selected to allow exploration of a variety of treatment and recovery pathways and include people with less common cancers, under-researched groups and groups with particular survivorship concerns.

\section{Inclusion criteria}

1. New diagnosis of one of the selected cancer types determined through clinical assessment, cytology, histology or imaging or new/second primary cancer at a site previously treated for cancer.

2. Awaiting primary curative intent treatment, including neoadjuvant treatment.

3. $\geq 16$ years old.

4. Able to complete questionnaires in English.

5. Able to provide written, informed consent.

\section{Exclusion criteria}

1. Disease recurrence/progression (either locally advanced or metastatic) at an existing cancer site.

2. Having treatment for a potentially curative recurrence of disease, for example, locally advanced disease (ie, they have been previously treated for the same cancer).

3. Metastatic disease from a cancer at another site (previous diagnosis of cancer at any other site would not be grounds for exclusion unless metastatic).

4. Synchronous primary cancers involving two or more of the HORIZONS specified cancer types.

Patients should be consented to HORIZONS prior to any treatment including surgery except for some ovarian and endometrial cancers where diagnosis is only confirmed at the time of surgery. These participants may 
enter the study following confirmed diagnosis. If a participant's status (eg, staging) changes after confirmed diagnosis they will be reassessed for eligibility. If later found to be ineligible, participants will be informed of this by a member of their clinical team. Study participants who develop metastases/disease progresses after consent will remain in the study.

\section{Assessment points}

$\mathrm{T}_{0}$ : baseline, following diagnosis and prior to initial curative intent treatment.

$\mathrm{T}_{1}: 3$ months after study entry to monitor early adaptation, coping and detect initial treatment effects.

$\mathrm{T}_{2}: 12$ months after study entry to monitor later adaptation, coping and detect initial treatment effects.

$\mathrm{T}_{3}$ : 18 months after study entry to monitor further adaptation and early stages of recovery.

$\mathrm{T}_{4}$ : 24 months after study entry to monitor consequences in the longer term and how they are managed.

$\mathrm{T}_{5}+$ : annual assessments to monitor consequences, emergence of late effects, comorbidities, lifestyle change and how they are experienced and managed across the life-course (subject to funding).

\section{Measures}

A selection of validated and study-specific outcome measures will be included, informed by the conceptual framework (figure 1), user and expert feedback. Key sociodemographic and socioeconomic information (eg, age, gender, ethincity, marital status, employment and so on) will be collected at baseline and characteristics that are likely to change will be included on a regular basis. Postcode data collected at consent will be used to calculate a measure of relative area deprivation: the Index of Multiple Deprivation. ${ }^{18}$ A core set of measures will be administered to all participants at each assessment point. Additional measures and questions will be included at different time points including burden of treatment, comorbidities, life events, lifestyle, social networks and the use of health and social care services. Selected measures (eg, the SF-12v2 ${ }^{19}$ ) will be incorportated to enable comparisions with the UK Understanding Society longtudinal household panel survey that captures social, economic and health data on the UK general population. ${ }^{20}$ All measures and questions assessed are described in supplementary appendix 2.

\section{Core outcome measures}

Quality of Life in Adult Cancer Survivors $(Q L A C S)^{21}$ will be the primary outcome measure. QLACS ${ }^{21}$ was developed to assess QoL of adult cancer survivors measuring 12 domains of cancer survivorship: generic (pain, fatigue, positive and negative feelings, cognitive and sexual problems, social avoidance) and cancer-specific (financial problems, family distress, recurrence distress, appearance concerns, benefits from cancer). QLACS has been validated among cancer survivors ${ }^{21}$ and has good convergent validity with other QoL measures (eg, Functional Assessment of Cancer Therapy and Short Form 36).

$E Q-5 D-5 L$ and the visual analogue scale $^{22} 23$ assesses health status for clinical and economic appraisal. It comprises of five domains: mobility, self-care, usual activities, pain/discomfort, anxiety/depression and five levels of severity. EQ-5D-5L has shown good measurement properties in a range of patient groups. ${ }^{24}$

EORTC-QLQ-C3O $O^{25}$ examines the impact of cancer and its treatment with 30 items assessing function (physical, role, cognitive, emotional and social), symptoms (eg, fatigue, pain, and nausea and vomiting) as well as global health and QoL.

EORTC site-specific modules will be included to capture disease-specific consequences: high grade NHL (NHL$\left.H G 29^{26}\right)$, breast $\left(B R 23^{27}\right)$, cervical $\left(C X 24^{28}\right)$, endometrial $\left(E N 24^{29}\right)$, ovarian $\left(O V 28^{30}\right)$ and vulval cancers $\left(V U 34^{31}\right)$. Site-specific modules will be supplemented with additional items from the EORTC item library ${ }^{32}$ to assess consequences not otherwise captured (eg, peripheral neuropathy, sexual function, menopausal symptoms).

Hospital Anxiety and Depression Scale (HADS $)^{33}$ comprises of 14 items with two 7-item subscales assessing anxiety and depression symptoms. HADS is used across a range of settings demonstrating good internal consistency, ${ }^{34}$ reliability and validity. ${ }^{35}$

Medical Outcomes Study Social Support Survey ${ }^{36}$ is a 19-item scale assessesing the level of social support available. It covers four domains (emotional/informational, tangible, affectionate support and positive social interaction) where higher scores indicate greater available support.

Self-efficacy for Managing Chronic Disease scale evaluates self-efficacy among pateints with chronic medical conditions. ${ }^{37}$ At baseline, the original 6-item version of the scale will be used and from 3 months onwards, an additional five cancer-specific items will be included forming the Cancer Survivors' Self-Efficacy Scale (CS-SES). The CS-SES has been demonstrated to have excellent reliability (Cronbach's alpha 0.92). ${ }^{38}$

Medical details: clinical information including cancer type, stage, treatment details, comorbid conditions, recurrence, relapse, survival, genetic tests, family history, route to diagnosis, follow-up care, height and weight, will be abstracted from clinical records by the participating centre. Change in health condition and treatment will be monitored through self-report at follow-up and further collection of clinical data from participating centres at regular intervals.

\section{Sample size}

Sample size calculations are based on the primary outcome measure. The mean generic summary score for QLACS is 71.2 (SD 25.6). ${ }^{21}$ A difference of one half of a SD is often quoted as a minimum clinically important difference in sample size calculations: using $80 \%$ power to detect a difference of one half of a SD at the 0.05 significance level would require a minimum of 228 participants per cancer type, assuming unequal-sized groups will be compared in 
the analyses. Assuming an intracluster correlation of 0.05 to allow for similarities between participants from each participating centre and an average of 30 participants per participating centre the cluster correction increases this figure to 559 per cohort. Taking into account $10 \%$ withdrawal and a range of 5-year survival rates according to cancer type and rounding up to allow for some subgroup analyses results in $\sim 1000$ participants per cohort. We are aiming for 3000 participants in total, although we anticipate around 750 DLBCL NHL patients. The sample size will be sufficient for other continuous measures, assuming the same size of difference (one half a SD). Subgroup analyses within cancer types will be possible, but numbers will be small for some instances, limiting the conclusions that can be drawn from these.

\section{Pilot phase}

During the pilot phase the HORIZONS Coordinating Centre will work with selected pilot participating centres to conduct feasibility work to assess study set up and training procedures, recruitment strategy, recruitment rates, representativeness of sample, appropriateness of measures for each of the tumour groups and clinical data. Pilot sites will be selected on the basis of geographical location, previous experience and hospital type.

\section{Procedure}

Patients will be identified at participating centres via a systematic screening process. Eligibility will be assessed using a standardised procedure and all eligible patients will be invited to participate over the recruitment period (September 2016 to March 2019). Eligible patients will, in general, be identified by a member of their direct care team through multidisciplinary team meetings or clinics. The HORIZONS Coordinating Centre will work with participating centres to review local procedures and determine best methods of screening and recruitment. Screening logs will be maintained at the participating centres. All eligible patients should be approached and invited to participate by a research nurse or member of the clinical team in the assessment clinic after their cancer diagnosis and before their first primary treatment. Participation in other research studies is not an exclusion criterion, unless it is an exclusion criterion of another study to which the participant is already consented. Patients who present as acute admissions (eg, emergency surgery) will be approached by their direct care team as soon as possible after surgery.

Participants will provide informed written consent to: participate (questionnaire completion and collection of medical details), be approached about related studies, for their data to be used in secondary data analyses and to be linked to UK national health databases. They will be given the opportunity to ask questions. They will then be given the baseline questionnaire to complete prior to their initial primary treatment which they will return directly to the HORIZONS Coordinating Centre along with their contact details form. Completed consent forms will be sent to the HORIZONS Coordinating Centre by participating sites. If participants require further time to consider participation, they may be given study information to take home and return to the HORIZONS Coordinating Centre. Research nurses at the participating centres may follow-up with a telephone call.

Follow-up questionnaires will be sent from the HORIZONS Coordinating Centre following status checks. Patients will be given the option of completing these on paper or online.

\section{Medical details CRF}

CRFs will be completed by the clinical team at baseline, 6 and 12 months and annually for all participants. Information including cancer type, stage, treatment, comorbid conditions, recurrence and survival will be recorded.

\section{Non-participation and withdrawal}

Participants will remain free to withdraw from HORIZONS at any time without giving a reason. Where possible reasons for non-participation will be recorded. If questionnaires have not been returned within 2 weeks, a reminder and duplicate questionnaire will be sent. If no response is received participants will not be approached again until the next follow-up questionnaire is due. Patients who decline any participation will be recorded on a decliners log containing aggregated data on age, gender, stage of disease and Eatern Cooperative Oncology Group (ECOG) performance status, in order to monitor the representativeness of HORIZONS. No identifiers of those who have declined will be held by the HORIZONS Coordinating Centre.

If, after consenting, a participant no longer meets the eligibility criteria they will be withdrawn from the study and their personal data and questionnaires destroyed. A member of the clinical team will inform the participant that they are no longer eligible for HORIZONS and will not be contacted again.

There are three withdrawal options:

1. No further contact: receive no further questionnaires but research team will retain and use information collected previously and to continue to access their medical records for research purposes.

2. No further access: receive no further questionnaires, research team to have no further access to their medical records, but information collected to date may be retained and used for research purposes.

3. No further use: receive no further questionnaires, research team to have no further access to their medical records and for information collected to date to be held only for regulatory purposes and not used for research purposes.

\section{Data quality}

The HORIZONS Coordinating Centre will be responsible for overseeing the handling and management of all study data. Paper copies of questionnaires and CRFs will be checked on receipt and any discrepancies or missing 
data queried. For specific questionnaire time points, participants will be contacted by the HORIZONS Coordinating Centre to complete missing items where possible to reduce missing data. Regular $10 \%$ data checks will be applied for data entry of paper questionnaires and CRFs into electronic databases. Electronic data will be routinely checked for accuracy.

\section{Measures to minimise bias}

At selection:

1. Patients identified through a systematic screening process.

2. Eligibility to participate assessed using a standardised procedure.

3. Non-identifiable data regarding reasons for non-participation will be collected.

4. Non-identifiable sociodemographic data will be collected (with verbal consent/agreement) on those who choose not to participate in the study.

5. Anonymised data collected for reasons why people were 'missed', that is, eligible but not invited to participate (eg, due to logistical reasons).

Outcome measurement and analysis:

1. All outcomes are self-rated.

2. Strenuous efforts will be made to reduce missing data and maintain participants in HORIZONS, including status checks to ensure participants have not moved or died, reminders being mailed on non-response and telephone calls to capture missing data.

\section{Data protection, data storage and patient confidentiality}

Data will be handled in compliance with Good Clinical Practice guidelines and data protection laws, following procedures laid out by the sponsor. This includes adherence to University of Southampton privacy and data protection policies, European and National regulations. All data will remain confidential at all times. Online data are hosted by a UK company which takes appropriate safety and security measures. All document repositories and databases are secured by various means including, but not limited to, user access by special controlled user IDs and passwords.

\section{Data-sharing plans}

Future data sharing will be subject to reasonable requests made in accordance with the Macmillan Survivorship Research Group (MSRG) data access policy. The MSRG data access policy and associated forms are available online at: https://www.horizons-hub.org.uk/access_data. html.

\section{Analysis}

\section{Recovery from cancer treatment}

Descriptive statistics will characterise the primary outcome of QoL (QLACS), describe health status (EQ-5D-5L) and symptoms/functioning (EORTC QLQ-C30 and site-specific modules). Changes in these outcomes over time will be investigated using longitudinal methods including generalised estimating equations. Group-based trajectory analyses will investigate whether there are distinct subgroups of recovery within each cohort. Factors that may influence recovery will be included in the regression models informed by the conceptual framework. The varying contribution of these factors will be assessed to determine which have the most influence on the outcomes of QoL, health status, well-being and symptoms/functioning following treatment. Recurrence and survival (overall and disease-specific) will be analysed using standard methods of survival analysis, such as Kaplan-Meier analysis.

\section{Self-managing the consequences of cancer}

Descriptive statistics will summarise participants' self-management activities, and their capacity and confidence to self-manage problems, and longitudinal analyses as previously described will assess changes over time in the measures of self-management (Health Education Impact Questionniare). ${ }^{39}$ Factors that may influence self-management will be investigated in the regression models informed by the conceptual framework. Analyses will explore how self-management practices relate to recovery of QoL, health and well-being. Self-management activities will be compared across groups including cancer and treatment types using statistical methods already described, and analyses such as group-based trajectory analyses will investigate whether there are distinct subgroups with different healthcare needs.

Patterns of missing data will be investigated in the analysis. Quantitative data will be analysed using statistical packages such as SPSS version 25 and Stata version 15.

\section{ETHICS}

Independent peer review of HORIZONS was coordinated by Macmillan Cancer Support in October 2013. HORIZONS has ethical and research governance approvals (IRAS Project ID: 202342, REC reference number 16/ NW/0425) and is adopted onto the National Institute for Health Research Clinical Research Network portfolio. University Hospital Southampton Foundation Trust is the Sponsor and will provide NHS to NHS indemnity. University Staff will be covered by the University of Southampton's Professional Indemnity and Clinical Trials Insurance for the conduct of the research.

We do not anticipate that there will be any risks to participants, but participants will be advised that, if they have any concerns about their health as a result of study participation, to contact their general practitioner (GP) or hospital doctor or nurse. The HORIZONS Coordinating Centre will provide details of support organisations. No individual response to questionnaire answers will be made by the HORIZONS Coordinating Centre, and participants will be made aware of this.

Protocol deviations will be documented and reported to the chief investigator (CI) and sponsor immediately. Steps will be taken to ensure questionnaires are sent out on time. Only serious adverse events (SAEs) during 
study participation deemed to be due to participation in HORIZONS will be reported to the CI within 24 hours of the participating centre learning of its occurrence. Local investigators will report any related SAEs as required by their local Research and Development Office.

\section{DISSEMINATION}

We will engage with our Scientific Advisory Board, Tumour Specific Expert Panels, User Reference Group, Macmillan Cancer Support and the University of Southampton to ensure maximum publicity and benefit. Findings will be widely disseminated through Macmillan Cancer Support, high impact publications, conference presentations and stakeholder events. We will make recommendations on where to target services and resources for cancer survivors. We will work with service users through our PPI events to disseminate study findings and discuss the progress of the study. HORIZONS has a dedicated website www.HORIZONS-hub.org.uk.

The full study dataset will be available to research staff within the HORIZONS Coordinating Centre. Other parties will only have access to the dataset, while the study is ongoing and the main results are being analysed and reported, where there are relevant agreements and permissions in place.

\section{STUDY MANAGEMENT}

The study will be delivered by the HORIZONS Coordinating Centre in the MSRG, University of Southampton, delegated this task by the sponsor. The study team is supported by a Programme Management Group (coapplicants), Strategic Advisory Board, User Reference Group, Tumour Specific Expert Panels and Data Monitoring Committee who are consulted and advise on various aspects of HORIZONS.

\section{SUMMARY}

The aims of HORIZONS are to:

1. Establish a series of cohorts of cancer patients to capture their health-related outcomes and experiences from beginning active treatment and regularly over time.

2. Maintain and develop HORIZONS as an accessible resource to explore consequences of different cancer diagnoses and treatments from the individual perspective.

3. Inform policy and practice-based innovative solutions to minimise health burden and maximise support available to people diagnosed with cancer.

The HORIZONS evidence will:

1. Improve understanding of the consequences of different cancers and treatments, the impacts of comorbidities, recurrence and late effects, the characteristics that lead to increased risk of poor recovery, the ability to self-manage and what helps or hinders this.
2. Support the transformation of personalised care for people living with and beyond cancer. Better information on short-term, medium-term and long-term outcomes and experiences across cancer types and treatments will enable health professionals to provide more personalised care to their patients tailored to their needs and to support people to live as healthy and active a life as possible.

3. Enable us to predict who is most likely to need support, what form this should take and when it should be available.

4. Help to prepare future patients for likely consequences and how long these might last following treatment so that they know what to look out for, when to seek support and to support decision-making where appropriate.

5. Identify areas for service innovation and other solutions to support cancer survivors and their personal networks to manage the consequences of cancer and its treatment across all aspects of their lives.

Acknowledgements We acknowledge the significant contributions of the following people in refining the HORIZONS protocol: Dr Joshua Turner and Dr Rebecca Foster (Research Fellows), Rebecca Petch and Amber Cole (Trial Coordinators), Professor Dame Jessica Corner (Chair of HORIZONS Strategic Advisory Board and advisor on original grant application), Professor Andy Davies (Chair of the NHL Tumour Specific Expert Panel), Professor Galina Velikova (Chair, Breast Tumour Specific Expert Panel), Dr Ros Glasspool (Chair, Gynaecological Tumour Specific Expert Panel) and their respective groups. With thanks to other members of the Macmillan Survivorship Research Group involved in protocol development.

Contributors CF conceptualised the project; $\mathrm{CF}$ (Cl) and coapplicants LC, AR, CRM, AR and PWS have developed the protocol. CF prepared and drafted the study protocol manuscript and received comments from coauthors. LC oversees the day-to-day running of the HORIZONS programme. All authors read and approved the final manuscript.

Funding The Macmillan HORIZONS Programme is funded by Macmillan Cancer Support (ref: 3546834) with a programme grant running from January 2016 to January 2021 and is sponsored by University Hospitals Southampton (ref: RHM CAN1199). The study protocol has received ethics and governance approvals from IRAS (ref: 202342). ClinicalTrials.gov Identifier: NC03000192.

Competing interests None declared.

Patient consent for publication Not required.

Provenance and peer review Not commissioned; externally peer reviewed.

Open access This is an open access article distributed in accordance with the Creative Commons Attribution Non Commercial (CC BY-NC 4.0) license, which permits others to distribute, remix, adapt, build upon this work non-commercially, and license their derivative works on different terms, provided the original work is properly cited, appropriate credit is given, any changes made indicated, and the use is non-commercial. See: http://creativecommons.org/licenses/by-nc/4.0/.

\section{REFERENCES}

1. Maddams J, Utley M, Møller H. Projections of cancer prevalence in the United Kingdom, 2010-2040. Br J Cancer 2012;107:1195-202.

2. May CR, Eton DT, Boehmer K, et al. Rethinking the patient: using Burden of Treatment Theory to understand the changing dynamics of illness. BMC Health Serv Res 2014;14:281.

3. Foster C, Fenlon D. Recovery and self-management support following primary cancer treatment. Br J Cancer 2011;105(Suppl 1):S21-8.

4. Corner J, Wright D, Hopkinson J, et al. The research priorities of patients attending UK cancer treatment centres: findings from a modified nominal group study. Br J Cancer 2007;96:875-81.

5. Foster $\mathrm{C}$, Wright $\mathrm{D}$, Hill $\mathrm{H}$, et al. Psychosocial implications of living 5 years or more following a cancer diagnosis: a systematic review of the research evidence. Eur J Cancer Care 2009;18:223-47. 
6. Jefford M, Karahalios E, Pollard A, et al. Survivorship issues following treatment completion-results from focus groups with Australian cancer survivors and health professionals. J Cancer Surviv 2008;2:20-32.

7. Hewitt M, Greenfield S, Stovall E. From cancer patient to cancer survivor: Lost in transition. Washington, DC: National Academies Press, 2005.

8. Armes J, Crowe M, Colbourne L, et al. Patients' supportive care needs beyond the end of cancer treatment: a prospective, longitudinal survey. J Clin Oncol 2009;27:6172-9.

9. Bell ML, Kenward MG, Fairclough DL, et al. Differential dropout and bias in randomised controlled trials: when it matters and when it may not. BMJ 2013;346:e8668.

10. Glaser AW, Fraser LK, Corner J, et al. Patient-reported outcomes of cancer survivors in England 1-5 years after diagnosis: a crosssectional survey. BMJ Open 2013;3:e002317.

11. Breckons M, Calman L, Foster CL. An online survey to examine cancer survivors' confidence to self-manage problems arising in the first 12 months following primary cancer treatment. In: Report MSRG, ed, 2012.

12. Santin $\mathrm{O}$, Mills $\mathrm{M}$, Treanor $\mathrm{C}$, et al. A comparative analysis of the health and well-being of cancer survivors to the general population. Support Care Cancer 2012;20:2545-52.

13. van de Poll-Franse LV, Horevoorts $\mathrm{N}$ vanE. The Patient Reported Outcomes Following Initial treatment and Long term Evaluation of Survivorship registry: Scope,rationale and design of an infrastructure for the study of physical and psychosocial outcomes in cancer survivorship cohorts. Eur J Cancer 2011.

14. Turner J, Kelly B, Clarke D, et al. A tiered multidisciplinary approach to the psychosocial care of adult cancer patients integrated into routine care: the PROMPT study (a cluster-randomised controlled trial). Supportive Care in Cancer 2017;25:17-26.

15. Gallacher K, May CR, Montori VM, et al. Understanding patients' experiences of treatment burden in chronic heart failure using normalization process theory. Ann Fam Med 2011;9:235-43.

16. Vassilev I, Rogers A, Blickem C, et al. Social networks, the 'work' and work force of chronic illness self-management: a survey analysis of personal communities. PLoS One 2013;8:e59723.

17. Vassilev I, Rogers A, Kennedy A, et al. The influence of social networks on self-management support: a metasynthesis. BMC Public Health 2014;14:719.

18. Office for National Statistics. https://www.gov.uk/government/ statistics/english-indices-of-deprivation-2015

19. Ware J, Kosinski M, Keller SD. A 12-Item Short-Form Health Survey: construction of scales and preliminary tests of reliability and validity. Med Care 1996;34:220-33.

20. University of Essex, Institute for Social and Economic Research. Understanding Society: Waves 1-8, 2009-2017 and Harmonised BHPS: Waves 1-18, 1991-2009. [data collection]. UK Data Service. 11th edn: SN, 2018:6614.

21. Avis NE, Smith KW, McGraw S, et al. Assessing quality of life in adult cancer survivors (QLACS). Qual Life Res 2005:14:1007-23.

22. EuroQol Group. EuroQol - a new facility for the measurement of health-related quality of life. Health Policy 1990;16:199-208.
23. Herdman M, Gudex C, Lloyd A, et al. Development and preliminary testing of the new five-level version of EQ-5D (EQ-5D-5L). Qual Life Res 2011;20:1727-36.

24. Janssen MF, Pickard AS, Golicki D, et al. Measurement properties of the EQ-5D-5L compared to the EQ-5D-3L across eight patient groups: a multi-country study. Qual Life Res 2013;22:1717-27.

25. Aaronson NK, Ahmedzai S, Bergman B, et al. The European Organization for Research and Treatment of Cancer QLQ-C30: a quality-of-life instrument for use in international clinical trials in oncology. J Natl Cancer Inst 1993;85:365-76.

26. van de Poll-Franse L, Oerlemans S, Bredart A, et al. International development of four EORTC disease-specific quality of life questionnaires for patients with Hodgkin lymphoma, high- and lowgrade non-Hodgkin lymphoma and chronic lymphocytic leukaemia. Qual Life Res 2018;27:333-45.

27. Sprangers MA, Groenvold M, Arraras JI, et al. The European Organization for Research and Treatment of Cancer breast cancerspecific quality-of-life questionnaire module: first results from a three-country field study. J Clin Oncol 1996;14:2756-68.

28. Greimel ER, Kuljanic Vlasic K, Waldenstrom AC, et al. The European Organization for Research and Treatment of Cancer (EORTC) Qualityof-Life questionnaire cervical cancer module: EORTC QLQ-CX24 Cancer 2006;107:1812-22.

29. Greimel E, Nordin A, Lanceley A, et al. Psychometric validation of the European Organisation for Research and Treatment of Cancer Quality of Life Questionnaire-Endometrial Cancer Module (EORTC QLQEN24). Eur J Cancer 2011;47:183-90.

30. Greimel E, Bottomley A, Cull A, et al. An international field study of the reliability and validity of a disease-specific questionnaire module (the QLQ-OV28) in assessing the quality of life of patients with ovarian cancer. Eur J Cancer 2003;39:1402-8.

31. Jensen P, Froeding L, Greimel E, et al. Development and pre-testing of the EORTC quality of life questionnaire for vulva cancer patients - the significance of patient involvement. Int J Gynecol Cancer 2017;27(S4):1770-70.

32. EORTC Item Library [ONLINE]. https://www.eortc.be/itemlibrary/

33. Zigmond AS, Snaith RP. The hospital anxiety and depression scale. Acta Psychiatr Scand 1983;67:361-70.

34. Moorey S, Greer S, Watson M, et al. The factor structure and factor stability of the hospital anxiety and depression scale in patients with cancer. Br J Psychiatry 1991;158:255-9.

35. Clark A, Fallowfield LJ. Quality of life measurements in patients with malignant disease: a review. J R Soc Med 1986;79:165-9.

36. Sherbourne CD, Stewart AL. The MOS social support survey. Soc Sci Med 1991:32:705-14.

37. Lorig KR, Ritter P, Stewart AL, et al. Chronic disease selfmanagement program: 2-year health status and health care utilization outcomes. Med Care 2001;39:1217-23.

38. Foster $\mathrm{C}$, Breckons $\mathrm{M}$, Hankins $\mathrm{M}$, et al. Developing a scale to measure self-efficacy to SELF-manage problems following cancer treatment. Psycho-Oncology 2013;22:16.

39. Osborne RH, Elsworth GR, Whitfield K. The Health Education Impact Questionnaire (heiQ): an outcomes and evaluation measure for patient education and self-management interventions for people with chronic conditions. Patient Educ Couns 2007;66:192-201. 